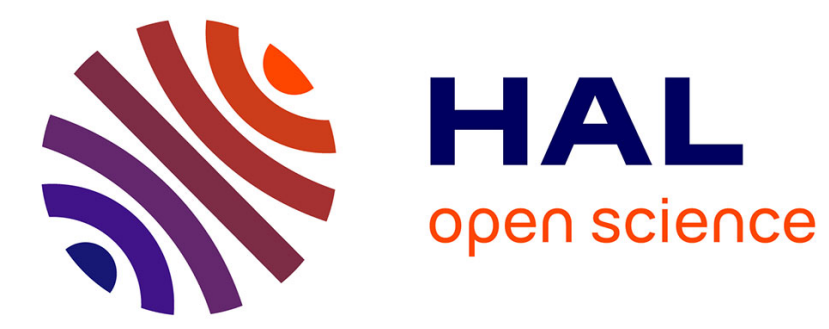

\title{
Porosity evolution in sintering systems
}

R. Glas, O. Blaschko, G. Krexner, P. Weinzierl

\section{To cite this version:}

R. Glas, O. Blaschko, G. Krexner, P. Weinzierl. Porosity evolution in sintering systems. Journal de Physique IV Proceedings, 1993, 03 (C8), pp.C8-345-C8-347. 10.1051/jp4:1993870 . jpa-00252301

\section{HAL Id: jpa-00252301 https://hal.science/jpa-00252301}

Submitted on 1 Jan 1993

HAL is a multi-disciplinary open access archive for the deposit and dissemination of scientific research documents, whether they are published or not. The documents may come from teaching and research institutions in France or abroad, or from public or private research centers.
L'archive ouverte pluridisciplinaire HAL, est destinée au dépôt et à la diffusion de documents scientifiques de niveau recherche, publiés ou non, émanant des établissements d'enseignement et de recherche français ou étrangers, des laboratoires publics ou privés. 


\title{
Porosity evolution in sintering systems
}

\author{
R. GLAS, O. BLASCHKO*, G. KREXNER* and P. WEINZIERL* \\ Laboratoire Léon Brillouin, CE Saclay, 91191 Gif-sur-Yvette, France \\ "Université Vienne, Strudlhofg. 4, 1090 Vienne, Austria
}

\begin{abstract}
Sintering in compacted Molybdenum powders is investigated using small angle neutron scattering. The scattering data permits to follow the evolution of the total pore surface $F$ together with the total pore volume $V$ in the course of the entire densification process. Characteristic changes in the relation between $F$ and $V$ suggest that, in particular, final stages of sintering (when the porosity can already be considered isolated from each other) are governed by a universal relation between $F$ and $V$ [1].
\end{abstract}

\section{Introduction}

The densification of a powder compact during sintering proceeds from a state characterized by a largely open porosity towards a system whose porosity is closed and isolated. With the gradual diminuation of the total void volume the sintered body approaches the limit of solid density.

The surface energy of the porosity constitutes the driving thermodynamic force that leads the systems to equilibrium and small angle scatttering in particular serves an excellent tool to follow the evolution of the total pore surface along with the total pore volume during the densification process. Different atomic transport mechanisms like surface, grain boundary and bulk diffusion, all of them participating to a varying extent in the elimination of the pore volume have been rendering a general theoretical description [2] of the change of density as a function of time and temperature rather complex.

\section{Experimental}

Two series of samples were prepared compacting Molybdenum powders of grain sizes of $3 \mu \mathrm{m}$ and $5 \mu m$ (labeled 'fine' and 'coarse' respectively) and sintering them at a number of temperatures for different times. The neutron small angle data were obtained on the $P A X E$ spectrometer at the Orphée reactor in Saclay with incident neutron wavelenghts between 5 and $8 \AA$. Sample thicknesses were kept as low as to ensure transmission values of above $90 \%$ which served to monitor the exclusion of multiple scattering contributions. The Porod [3] dependence $\left(I \propto F / Q^{4}\right)$ of the intensity distributions obtained served to determine the total pore surface the total pore volume being determined via the macroscopic density.

\section{Results}

The surface values $F$ as a function of the pore volume fraction $V$ obtained for the different sintering treatments are shown in Fig.1. For reasons becoming evident below the square root of the pore surface is chosen as the ordinate of the plot. The inset gives the time evolution of the density of the coarse-grained powder for the three temperatures investigated i.e. $1500^{\circ} \mathrm{C}, 2000^{\circ} \mathrm{Cand} 2500^{\circ} \mathrm{C}$. Despite the fact that the density evolution takes a clearly different course depending on temperature the ensemble of coarse-grained molybdenum samples is represented by a single straight line in the $\sqrt{F}-V$ plot (solid line). The data points can be described to a good approximation by the equation $F=a \cdot V^{2}$ and this relation holds regardless of the time and temperature parameters of the sintering treatment. The constant $a$, however, shows to be somewhat different for the two grain sizes investigated and turns out to depend on the initial $(F, V)$ characteristics of the powder 
compact. In the beginning the sintering system composed of the finer powder shows a smaller total cavity volume but a similar pore surface compared to the coarse-grained powder system and hence results in a steeper descent although it can be likewise represented by a $F=a \cdot V^{2}$ relation (dashed line). Although the accuracy of the pore volume fractions determined decreases by decreasing pore volume the dominating signature of the $F \sim V^{2}$ trajectory is sustained by the data points corresponding to higher pore volume fractions where the uncertainties are low.

\section{Discussion}

The consequences of the $F \sim V^{2}$ behaviour observed are to be discussed based on the frequently used assumption that the ensemble of pores can reasonably well be described by a characteristic pore radius $R$. Hence, apart from geometrical factors, $F=V / R$ and the two mechanisms for a change of $F$ are:

a) $d F=d V / R$ describing a reduction of the pore surface solely by reduction of the pore volume with the pore size $R$ remaining constant which results in a linear relation between $F$ and $V$ as it occurs due to a pure evaporation of the porosity at the macroscopic surface of the sample.

b) $d F=-\left(V / R^{2}\right) d R$ describing a reduction of the pore surface solely by a coarsening of the pores at constant pore volume.

From the observed quadratic relation between $F$ and $V$ it follows that the integral cavity surface is more strongly reduced than the total cavity volume. This means that in addition to pore elimination pore coarsening occurs during this stage of densification and that both of the above mechanisms contribute to the sintering process.

Equating the differential of the experimental finding, $d F / F=2 d V / V$, and the differential of the general relation $(F=V / R), d F / F=d V / V-d R / R$ one yields $d V / V=-d R / R$. This illustrates that the variation of both variables, $R$ and $V$, contributes to the evolution of the total pore surface. Moreover their relative variation is equal which shows that both mechanisms cited above are active to the same extent in order to reduce the total pore surface.

This remarkable consequence is further illustrated in Fig. 2 where the experimental results are presented in a $(1 / R, V)$ plot. In agreement with the ident relative variation of $R$ and $V$ the data points follow a straight line through the origin. This means that starting from $(F, V)$ values depending on the respective initial powder characteristics all systems develop in the most direct way, i.e. on straight lines, towards their thermodynamic equilibrium configuration represented by the origin of the $(1 / R, V)$ plot.

Compared to the coarse powder compacts investigations on the finer powder samples indicated that freshly prepared compacts of finer powders not necessarily fall on the straight line of the later $F \sim V^{2}$ trajectory. Preliminary results on the very early stages of these finer powders indicate that the final stage is preceded by a first rounding off of the cusped voids that reduces the inner surface of the porosity but leaves the volume untouched and therefore no densification takes place. A transitory regime during which a degradation of the connectivity of the porosity takes place marks the onset of densification. The extension, however, of this joint between the initial stage and the situation where the porosity is yet isolated depends strongly on the production of the powder compact (pressure and powder grain size).

The concept of equally present evaporation and coarsening mechanisms in the late stages of densification explains the observed evolution of the sintering systems however standard theories of coarsening $[4,5]$ do not apply as manifested by the $F \sim V^{2}$ relation. This cannot be expected on the other hand as an essential assumption of the LSW theory, the conservation of volume, is not met by the sintering process as well as the different diffusion mechanism taking part in the evolution are not incorporated in the LSW theory.

\section{Conclusion}

We have tried to establish a detailed description of the surface evolution of a standard sintering system and for the final stages we observed a universal $F \sim V^{2}$ behaviour that holds regardless of the sintering parameters. Consequently both mechanisms, coarsening and pore evaporation at the macroscopic surface, must take part in the reduction of the surface energy and the two processes likewise contribute to the progress of the system towards thermodynamic equilibrium. For the initial and intermediate stages there is evidence of characteristic changes in the dependence of $F$ on $V$. However, further work is necessary in order to deepen the understanding of the functional relationship between these quantities also for the earlier stages of the sintering process. 


\section{References}

[1] O.Blaschko, R.Glas, G.Krexner and P.Weinzierl, Phys.Rev.Lett. 68, 970 (1992).

[2] M.F.Ashby, Acta metall. 22, 275 (1974);

F.B.Swinkels and M.F. Ashby, Acta metall. 29, 259 (1981).

[3] G. Porod, Kolloid-Zs. 124, 83 (1951).

[4] I.M. Lifshitz and V.V.Slyozov, J.Phys.Chem.Solids 19, 35 (1961).

[5] C.Wagner, Z.Elektrochem. 65, 581 (1961).

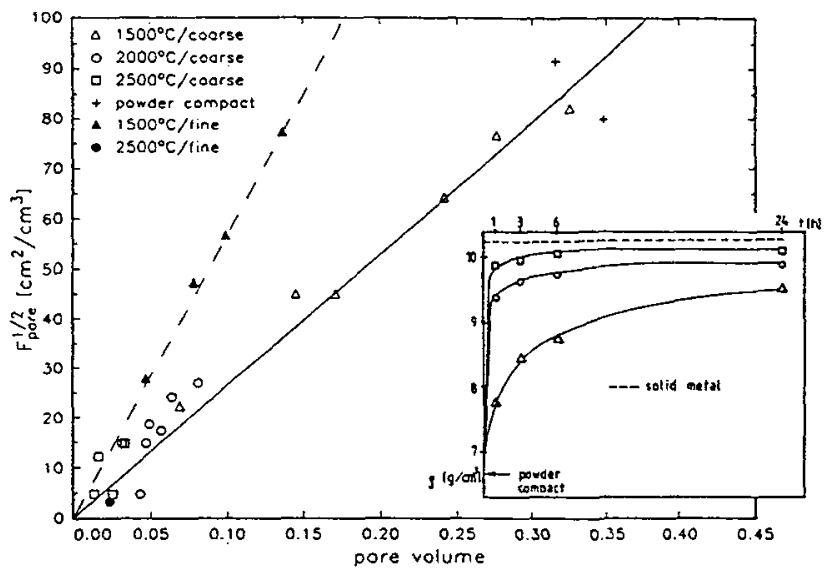

Fig.t : The sintering process in molybdenum: Evolution of the square root of the total pore surface area $F$ as a function of the total pore volume $V . V$ is given as the sample volume fraction occupied by voids. Sintering times cover a range from 10 minutes 1024 hours. The straight lines are guides to the eye (solid line: coarse-grained powder. dashed line: finegrained powder). The inset shows three curves giving the time dependence of the density of the coarse-grained powder for three different sintering temperatures. The melting point of molybdenum is $2617^{\circ} \mathrm{C}$.

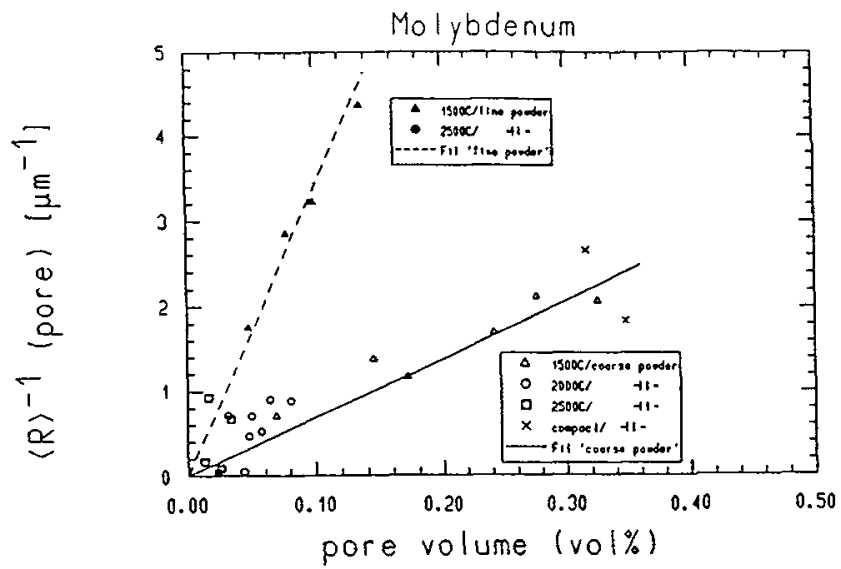

Fig. 2 : Sintering trajectories for the two molybdenum powders in a $(1 / R, V)$ representation. The straight lines show that $R$ and $V$ exhibit (except for their sign) the same relative variation. 